\title{
Reconstruction formulas for Photoacoustic Imaging in Attenuating Media
}

\author{
Otmar Scherzer ${ }^{1,2}$ \\ otmar.scherzer@univie.ac.at
}

\author{
Cong Shi ${ }^{1}$ \\ cong.shi@univie.ac.at
}

May 23, 2017

${ }^{1}$ Computational Science Center

University of Vienna

Oskar-Morgenstern-Platz 1

A-1090 Vienna, Austria

\author{
${ }^{2}$ Johann Radon Institute for Compu- \\ tational and Applied Mathematics \\ (RICAM)
}

Altenbergerstraße 69

A-4040 Linz, Austria

\begin{abstract}
In this paper we study the problem of photoacoustic inversion in a weakly attenuating medium. We present explicit reconstruction formulas in such media and show that the inversion based on such formulas is moderately ill-posed. Moreover, we present a numerical algorithm for imaging and demonstrate in numerical experiments the feasibility of this approach.
\end{abstract}

\section{Introduction}

When a probe is excited by a short electromagnetic (EM) pulse, it absorbs part of the EM-energy, and expands as a reaction, which in turn produces a pressure wave. In photoacoustic experiments, using measurements of the pressure wave, the ability of the medium to transfer absorbed EM-energy into pressure waves is visualized and used for diagnostic purposes. Common visualizations, see [26], are based on the assumptions that the specimen can be uniformly illuminated, is acoustically non-attenuating, and that the sound-speed and compressibility are constant.

In mathematical terms, the photoacoustic imaging problem consists in calculating the compactly supported absorption density function $h: \mathbb{R}^{3} \rightarrow$ 
$\mathbb{R}$, appearing as a source term in the wave equation

$$
\begin{aligned}
\partial_{t t} p(t, \mathbf{x})-\Delta p(t, \mathbf{x}) & =\delta^{\prime}(t) h(\mathbf{x}), & & t \in \mathbb{R}, \mathbf{x} \in \mathbb{R}^{3}, \\
p(t, \mathbf{x}) & =0, & & t<0, \mathbf{x} \in \mathbb{R}^{3}
\end{aligned}
$$

from some measurements over time of the pressure $p$ on a two-dimensional manifold $\Gamma$ outside of the specimen, that is outside of the support of the absorption density function. This problem has been studied extensively in the literature (see e.g. [14, 25, 13], to mention just a few survey articles).

Biological tissue has a non-vanishing viscosity, thus there is thermal consumption of energy. These effects can be described mathematically by attenuation. Common models of such are the thermo-viscous model [9], its modification [11], Szabo's power law [22, 21] and a causal modification [10], Hanyga \& Seredy'nska [5], Sushilov \& Cobbold [20], and the NachmanSmith-Waag model [15]. Photoacoustic imaging in attenuating medium then consists in computing the absorption density function $h$ from measurements of the attenuated pressure $p^{a}$ on a surface containing the object of interest. The attenuated pressure equation reads as follows

$$
\begin{aligned}
\mathcal{A}_{\kappa}\left[p^{a}\right](t, \mathbf{x})-\Delta p^{a}(t, \mathbf{x}) & =\delta^{\prime}(t) h(\mathbf{x}), & & t \in \mathbb{R}, \mathbf{x} \in \mathbb{R}^{3}, \\
p^{a}(t, \mathbf{x}) & =0, & & t<0, \mathbf{x} \in \mathbb{R}^{3},
\end{aligned}
$$

where $\mathcal{A}_{\kappa}$ is the pseudo-differential operator defined in frequency domain (see (1.4)). The formal difference between (1.1) and (1.2) is that the second time derivative operator $\partial_{t t}$ is replaced by a pseudo-differential operator $\mathcal{A}_{\kappa}$.

The literature on Photoacoustics in attenuating media concentrates on time-reversal and attenuation compensation based on power laws: We mention the $k$-wave toolbox implementation and the according papers [3, 24], [2, 7]. In [4] several attenuation laws from the literature have been cataloged into two classes, namely weak and strong attenuation laws. Power laws lead, in general, to severely ill-posed photoacoustic imaging problem, while mathematically sophisticatedly derived models, like the Nachman-Smith-Waag model [15], lead to moderately ill-posed problems.

The paper is based on the premise that Photoacoustics is moderately illposed, and we therefore concentrate on photoacoustic inversion in weakly attenuating media, which have not been part of extensive analytical and numerical studies in the literature. Another goal of this work is to derive explicit reconstruction formulas for the absorption density function $h$ in attenuating media. Previously there have been derived asymptotical expansions in the case of small absorbers $[1,8]$. 


\section{Notation}

We use the following notations:

- For $s=0,1,2, \ldots$ we denote by $\mathcal{S}\left(\mathbb{R} \times \mathbb{R}^{s}\right)$ the Schwartz-space of complex valued functions and its dual space, the space of tempered distribution, is denoted by $\mathcal{S}^{\prime}\left(\mathbb{R} \times \mathbb{R}^{s}\right)$.

We abbreviate $\mathcal{S}=\mathcal{S}\left(\mathbb{R} \times \mathbb{R}^{3}\right)$ and $\mathcal{S}^{\prime}=\mathcal{S}^{\prime}\left(\mathbb{R} \times \mathbb{R}^{3}\right)$.

- For $\phi \in \mathcal{S}(\mathbb{R})$ we define the Fourier-transform by

$$
\hat{\phi}(\omega)=\frac{1}{\sqrt{2 \pi}} \int_{t=-\infty}^{\infty} e^{\mathrm{i} \omega t} \phi(t) \mathrm{d} t,
$$

and the one-dimensional inverse Fourier-transform is given by

$$
\check{\varphi}(t)=\frac{1}{\sqrt{2 \pi}} \int_{\omega=-\infty}^{\infty} e^{-\mathrm{i} \omega t} \varphi(\omega) \mathrm{d} \omega .
$$

- Let $\varphi \in \mathcal{S}(\mathbb{R})$ and $\psi \in \mathcal{S}\left(\mathbb{R}^{3}\right)$. The Fourier-transform $\mathcal{F}[\cdot]: \mathcal{S}^{\prime} \rightarrow \mathcal{S}^{\prime}$ on the space of tempered distributions is defined by

$$
\langle\mathcal{F}[u], \varphi \otimes \psi\rangle_{\mathcal{S}^{\prime}, \mathcal{S}}=\langle u, \check{\varphi} \otimes \psi\rangle_{\mathcal{S}^{\prime}, \mathcal{S}}
$$

Note that for functions $u \in \mathcal{S}$ we have

$$
\langle\mathcal{F}[u], \varphi \otimes \psi\rangle_{\mathcal{S}^{\prime}, \mathcal{S}}=\int_{\mathbb{R} \times \mathbb{R}^{3}} u(t, \mathbf{x} \overline{\check{\varphi}(t) \psi(\mathbf{x})} \mathrm{d} t \mathrm{~d} \mathbf{x} .
$$

We are identifying distributions and functions and we are writing in the following for all $u \in \mathcal{S}^{\prime}$

$$
\begin{aligned}
\mathcal{F}[u](\omega, \mathbf{x}) & =\frac{1}{\sqrt{2 \pi}} \int_{t=-\infty}^{\infty} e^{\mathrm{i} \omega t} u(t, \mathbf{x}) \mathrm{d} t \text { and } \\
\mathcal{F}^{-1}[y](t, \mathbf{x}) & =\frac{1}{\sqrt{2 \pi}} \int_{\omega=-\infty}^{\infty} e^{-i \omega t} y(\omega, \mathbf{x}) \mathrm{d} \omega .
\end{aligned}
$$


- We define the attenuation operator $\mathcal{A}_{\kappa}[\cdot]: \mathcal{S}^{\prime} \rightarrow \mathcal{S}^{\prime}$ by

$$
\left\langle\mathcal{A}_{\kappa}[u], \phi \otimes \psi\right\rangle_{\mathcal{S}^{\prime}, \mathcal{S}}=-\left\langle u, \overline{\kappa^{2} \hat{\phi}} \otimes \psi\right\rangle_{\mathcal{S}^{\prime}, \mathcal{S}} .
$$

This means that if $u \in \mathcal{S}\left(\mathbb{R} \times \mathbb{R}^{3}\right)$ then

$$
\mathcal{A}_{\kappa}[u](t, \mathbf{x})=-\mathcal{F}^{-1}\left[\kappa^{2} \mathcal{F}[u]\right](t, \mathbf{x}), \quad \omega \in \mathbb{R}, \mathbf{x} \in \mathbb{R}^{3} .
$$

- $\mathcal{I}[\cdot]$ denotes the time integration operator on the space of tempered distributions and is given by

$$
\langle\mathcal{I}[u], \phi \otimes \psi\rangle_{\mathcal{S}^{\prime}, \mathcal{S}}=-\left\langle u, \phi^{\prime} \otimes \psi\right\rangle_{\mathcal{S}^{\prime}, \mathcal{S}},
$$

and we write formally for $u \in \mathcal{S}^{\prime}$

$$
u \rightarrow \mathcal{I}[u](t, \mathbf{x})=\int_{-\infty}^{t} u(\tau, \mathbf{x}) d \tau
$$

- (1.2) and (1.1) (here $\kappa(\omega)=\omega^{2}$ ) are understood in a distributional sense, which means that for all $\phi \in \mathcal{S}(\mathbb{R})$ and $\psi \in \mathcal{S}\left(\mathbb{R}^{3}\right)$

$$
\begin{aligned}
& \left\langle p^{a}, \overline{\kappa^{2}} \hat{\phi} \otimes \psi\right\rangle_{\mathcal{S}^{\prime}, \mathcal{S}}+\left\langle p^{a}, \phi \otimes \Delta_{\mathbf{x}} \psi\right\rangle_{\mathcal{S}^{\prime}, \mathcal{S}} \\
= & \phi^{\prime}(0)\langle h, \psi\rangle_{\mathcal{S}^{\prime}\left(\mathbb{R}^{3}\right), \mathcal{S}\left(\mathbb{R}^{3}\right)} .
\end{aligned}
$$

\section{Attenuation}

Attenuation describes the physical phenomenon that certain frequency components of acoustic waves are attenuated more rapidly over time. Mathematically this is encoded in the function $\kappa$ defining the pseudo-differential operator $\mathcal{A}_{\kappa}$. A physically and mathematically meaningful $\kappa$ has to satisfy the following properties (see [4]):

Definition 2.1 We call a non-zero function $\kappa \in C^{\infty}(\mathbb{R} ; \overline{\mathbb{H}})$, where $\mathbb{H}=$ $\{z \in \mathbb{C}: \Im \mathrm{m} z>0\}$ denotes the upper half complex plane and $\overline{\mathrm{H}}$ its closure in $\mathbb{C}$, an attenuation coefficient if

1. all the derivatives of $\kappa$ are polynomially bounded. That is, for every $\ell \in \mathbb{N}_{0}$ there exist constants $\kappa_{1}>0$ and $N \in \mathbb{N}$ such that

$$
\left|\kappa^{(\ell)}(\omega)\right| \leq \kappa_{1}(1+|\omega|)^{N}
$$


2. There exists a holomorphic continuation $\tilde{\kappa}: \overline{\mathbb{H}} \rightarrow \overline{\mathrm{H}}$ of $\kappa$ on the upper half plane, that is, $\tilde{\kappa} \in C(\overline{\mathbb{H}} ; \overline{\mathrm{H}})$ with $\left.\tilde{\kappa}\right|_{\mathbb{R}}=\kappa$ and $\tilde{\kappa}: \mathbb{H} \rightarrow \overline{\mathbb{H}}$ is holomorphic, and there exists constants $\tilde{\kappa}_{1}>0$ and $\tilde{N} \in \mathbb{N}$ such that

$$
|\tilde{\kappa}(z)| \leq \tilde{\kappa}_{1}(1+|z|)^{\tilde{N}} \quad \text { for all } \quad z \in \overline{\mathrm{H}}
$$

3. $\kappa$ is symmetric: That is, $\kappa(-\omega)=-\overline{\kappa(\omega)}$ for all $\omega \in \mathbb{R}$.

4. There exists some constant $c>0$ such that the holomorphic extension $\tilde{\kappa}$ of the attenuation coefficient $\kappa$ satisfies

$$
\Im \mathrm{m}\left(\tilde{\kappa}(z)-\frac{z}{c}\right) \geq 0 \quad \text { for every } z \in \overline{\mathrm{H}} \text {. }
$$

Remark: The four conditions in Definition 2.1 on $\kappa$ encode the following physical properties of the attenuated wave equation (see [4]):

The condition (2.1) in Definition 2.1 ensures that the product $\kappa^{2} u$ of $\kappa^{2}$ with an arbitrary tempered distribution $u \in \mathcal{S}^{\prime}$ is again in $\mathcal{S}^{\prime}$ and therefore, the operator $\mathcal{A}_{\kappa}: \mathcal{S}^{\prime} \rightarrow \mathcal{S}^{\prime}$ is well-defined on the space of tempered distributions.

The second condition guarantees that the solution of the attenuated wave equation is causal.

Condition three ensures that real valued distributions (such as the pressure) are mapped to real valued distributions. That is $p^{a}$ is real valued if the absorption density $h$ is real.

The forth condition guarantees that the solution $p^{a} \in \mathcal{S}^{\prime}\left(\mathbb{R} \times \mathbb{R}^{3}\right)$ of the equation (1.2) propagates with finite speed $c>0$. That is

$$
\operatorname{supp} p^{a} \subset\left\{(t, x) \in \mathbb{R} \times \mathbb{R}^{3}:|x| \leq c t+R\right\}
$$

whenever $\operatorname{supp} h \subset B_{R}(0)$.

In the literature there have been documented two classes of attenuation models:

Definition 2.2 We call an attenuation coefficient $\kappa \in C^{\infty}(\mathbb{R} ; \overline{\mathbb{H}})$ (see Definition 2.1) 
- a weak attenuation coefficient if there exists a constant $0 \leq k_{\infty} \in \mathbb{R}$ and a bounded function $k_{*} \in C^{\infty}(\mathbb{R} ; \mathbb{C}) \cap L^{2}(\mathbb{R} ; \mathbb{C})$ such that

$$
\kappa(\omega)=\omega+\mathrm{i} k_{\infty}+k_{*}(\omega) \text { for all } \omega \in \mathbb{R} .
$$

In particular, $\kappa$ is constantly attenuating, if $\kappa$ is a weak attenuation coefficient with $k_{*} \equiv 0$. That is, there exists a constant $k_{\infty} \geq 0$ such that

$$
\kappa(\omega)=\omega+\mathrm{i} k_{\infty} \text { for all } \omega \in \mathbb{R} .
$$

- $\kappa$ is called strong attenuation coefficient if there exist constants $\kappa_{0}>0$, $\beta>0$, and $\omega_{0}>0$

$$
\Im \mathrm{m} \kappa(\omega) \geq \kappa_{0}|\omega|^{\beta} \text { for all } \omega \in \mathbb{R} \text { with }|\omega| \geq \omega_{0} .
$$

For such attenuation coefficients we proved in [4] well-posedness of the attenuated wave equation:

Lemma 2.3 Let $\kappa$ be an attenuation coefficient. Then the solution $p^{a}$ of the equation (1.2) exists and is a real-valued tempered distribution in $\mathbb{R} \times \mathbb{R}^{3}$.

Moreover, $q^{a}:=\mathcal{I}\left[p^{a}\right]$ is a tempered distribution and satisfies the equation

$$
\begin{aligned}
\mathcal{A}_{\kappa}\left[q^{a}\right](t, \mathbf{x})-\Delta q^{a}(t, \mathbf{x}) & =\delta(t) h(\mathbf{x}), & & t \in \mathbb{R}, \mathbf{x} \in \mathbb{R}^{3}, \\
q^{a}(t, \mathbf{x}) & =0, & & t<0, \mathbf{x} \in \mathbb{R}^{3},
\end{aligned}
$$

and in Fourier domain

$$
\kappa^{2}(\omega) \mathcal{F}\left[q^{a}\right](\omega, \mathbf{x})+\Delta_{\mathbf{x}} \mathcal{F}\left[q^{a}\right](\omega, \mathbf{x})=-\frac{1}{\sqrt{2 \pi}} h(\mathbf{x}) .
$$

Proof: The first item has been proven in [4]. The second item is an immediate consequence of the definition of a tempered distribution.

Remark: (2.5) has to be understood in a distributional sense: That is $q^{a} \in \mathcal{S}^{\prime}$ and satisfies for every $\phi \in \mathcal{S}(\mathbb{R})$ and $\psi \in \mathcal{S}\left(\mathbb{R}^{3}\right)$ the equation

$$
\left\langle\mathcal{A}_{\kappa}\left[q^{a}\right], \phi \otimes \psi\right\rangle_{\mathcal{S}^{\prime}, \mathcal{S}}-\left\langle q^{a}, \phi \otimes \Delta_{\mathbf{x}} \psi\right\rangle_{\mathcal{S}^{\prime}, \mathcal{S}}=\phi(0)\langle h, \psi\rangle_{\mathcal{S}^{\prime}\left(\mathbb{R}^{3}\right), \mathcal{S}\left(\mathbb{R}^{3}\right)} .
$$

If $\kappa(\omega)=\omega$ (that is the case of the standard wave equation) $q=q^{a}$ solves the following equation in a distributional sense

$$
\begin{aligned}
\partial_{t t} q(t, \mathbf{x})-\Delta_{\mathbf{x}} q(t, \mathbf{x}) & =\delta(t) h(\mathbf{x}), & & t \in \mathbb{R}, \mathbf{x} \in \mathbb{R}^{3}, \\
q(t, \mathbf{x}) & =0, & & t<0, \mathbf{x} \in \mathbb{R}^{3},
\end{aligned}
$$


and its Fourier-transform $\mathcal{F}[q]$ satisfies the Helmholtz equation

$$
\omega^{2} \mathcal{F}[q](\omega, \mathbf{x})+\Delta_{\mathbf{x}} \mathcal{F}[q](\omega, \mathbf{x})=-\frac{1}{\sqrt{2 \pi}} h(\mathbf{x}), \quad \omega \in \mathbb{R}, \mathbf{x} \in \mathbb{R}^{3} .
$$

Again $q \in \mathcal{S}^{\prime}\left(\mathbb{R} \times \mathbb{R}^{3}\right)$ and satisfies (2.8) in a distributional sense:

$$
\left\langle q, \partial_{t t} \phi \otimes \psi\right\rangle_{\mathcal{S}^{\prime}, \mathcal{S}}-\left\langle q, \phi \otimes \Delta_{\mathbf{x}} \psi\right\rangle_{\mathcal{S}^{\prime}, \mathcal{S}}=\phi(0)\langle h, \psi\rangle_{\mathcal{S}^{\prime}\left(\mathbb{R}^{3}\right), \mathcal{S}\left(\mathbb{R}^{3}\right)} .
$$

The solution of (2.8) can also be written as the solution of the initial value problem:

$$
\begin{aligned}
\partial_{t t} q(t, \mathbf{x})-\Delta_{\mathbf{x}} q(t, \mathbf{x}) & =0, & & t>0, \mathbf{x} \in \mathbb{R}^{3}, \\
q(0, \mathbf{x}) & =0, & & \mathbf{x} \in \mathbb{R}^{3}, \\
\partial_{t} q(0, \mathbf{x}) & =h(\mathbf{x}), & & \mathbf{x} \in \mathbb{R}^{3} .
\end{aligned}
$$

In the following we derive a functional relation between $q$ and $q^{a}$, which is the basis of analytical back-projection formulas in attenuating media.

Theorem 2.4 Let $\phi \in \mathcal{S}(\mathbb{R})$ and define

$$
\nu_{+}[\phi](\tau):=\frac{1}{\sqrt{2 \pi}} \int_{\omega=-\infty}^{\infty} e^{-\mathrm{i} \overline{\kappa(\omega)} \tau} \hat{\phi}(\omega) \mathrm{d} \omega \text { for all } \tau \geq 0 .
$$

Then there exists a sequence $\left(a_{m}\right)_{m \geq 1}$ of real numbers satisfying $\sum_{m \geq 1} a_{m} 2^{m j}=$ $(-1)^{j}$ and a function $\vartheta \in C_{0}^{\infty}(\mathbb{R} ; \mathbb{R})$ such that $\vartheta(\tau)=1$ when $|\tau|<1$ and $\vartheta(\tau)=0$ when $|\tau| \geq 2$ such that the function $\nu: \mathbb{R} \rightarrow \mathbb{C}$, defined by

$$
\nu(\tau):=\nu[\phi](\tau):= \begin{cases}\nu_{+}[\phi](\tau) & \text { for all } \quad \tau \geq 0 \\ \sum_{m=0}^{\infty} a_{m} \nu_{+}[\phi]\left(-2^{m} \tau\right) \vartheta\left(-2^{m} \tau\right) & \text { for all } \quad \tau<0\end{cases}
$$

is an element of the Schwartz space $\mathcal{S}(\mathbb{R})$.

Proof: $\quad$ 1. Using that for all $k \in \mathbb{N}_{0}$

$$
\psi_{k}(\tau, \omega):=\partial_{\tau}^{k} e^{-\mathrm{i} \overline{\kappa(\omega)} \tau}=(-\mathrm{i})^{k} \overline{\kappa(\omega)} k e^{-\mathrm{i} \overline{\kappa(\omega)} \tau} \text { for all } \omega, \tau \in \mathbb{R},
$$

it follows from Definition 2.2 that, uniformly in $\tau$, for all $\omega \in \mathbb{R}$

$$
\left|\psi_{k}(\tau, \omega) \hat{\phi}(\omega)\right| \leq|\kappa(\omega)|^{k} e^{-\Im m \kappa(\omega) \tau}|\hat{\phi}(\omega)| \leq|\kappa(\omega)|^{k}|\hat{\phi}(\omega)| .
$$


From $(2.1)$ and $\hat{\phi} \in \mathcal{S}(\mathbb{R})$ (in particular $\kappa \in L^{\infty}(\mathbb{R} ; \mathbb{C}$ ) and $\hat{\phi} \in$ $C(\mathbb{R} ; \mathbb{C})), \omega \rightarrow|\kappa(\omega)|^{k}|\hat{\phi}(\omega)| \in L^{1}(\mathbb{R} ; \mathbb{R})$. Thus by interchanging integration and differentiation it follows that for $R \rightarrow \infty$

$$
d^{k} \nu_{+}[\phi](\tau)=\frac{1}{\sqrt{2 \pi}} \int_{\omega=-\infty}^{\infty} \psi_{k}(\tau, \omega) \hat{\phi}(\omega) \mathrm{d} \omega \text { for all } \tau \geq 0
$$

and that these functions are continuous. Thus $\nu_{+}[\phi] \in C^{\infty}([0, \infty) ; \mathbb{C})$.

2. From [18] it follows that $\nu[\phi]$ defined in $(2.12)$ is in $C^{\infty}(\mathbb{R} ; \mathbb{C})$ and extends the function $\nu_{+}[\phi]$ defined on $[0, \infty)$.

3. We are proving that $\nu_{+}[\phi]$ and all its derivative are faster decaying than polynomials in $\tau$ for $\tau \rightarrow+\infty$.

For this purpose we use the stationary phase method summarized in Theorem A.1:

Let $\theta \in C_{0}^{\infty}(\mathbb{R} ; \mathbb{R})$ be a mollifier satisfying $\theta(\omega)=1$ when $|\omega|<1$ and $\theta(\omega)=0$ when $|\omega| \geq 2$. For $k \in \mathbb{N}$ and $R>0$ fixed, we apply the stationary phase method with

$$
\omega \rightarrow f(\omega)=-\overline{\kappa(\omega)} \text { and } \omega \rightarrow g_{R}(\omega):=\psi_{k}(\tau, \omega) \theta(\omega / R) .
$$

Below we are verifying the assumptions of the stationary phase method:

- $f \in C^{\infty}(\mathbb{R} ; \mathbb{C})$ by Definition 2.1 and $g_{R} \in C_{0}^{\infty}(\mathbb{R} ; \mathbb{C})$, because it is the product of a $C^{\infty}(\mathbb{R} ; \mathbb{C})$ function and the compactly supported function $\omega \rightarrow \theta(\omega / R)$.

- The second property

$$
\Im \mathrm{m} f=\Im \mathrm{m} \kappa \geq 0 .
$$

is an immediate consequence of the assumption $\kappa \in C^{\infty}(\mathbb{R} ; \overline{\mathbb{H}})$ in Definition 2.1.

Thus (A.1) can be applied with the functions $f=-\bar{\kappa}$ and $g_{R}$, and 
using Lemma A.2 (A.2) it follows that

$$
\begin{aligned}
& \tau^{l}\left|\int_{\omega=-\infty}^{\infty} e^{\mathrm{i} \tau f(\omega)} g_{R}(\omega) \mathrm{d} \omega\right| \\
& \leq C_{1} \sum_{\alpha=0}^{l} \sup _{\omega \in \mathbb{R}}\left|d^{\alpha} g_{R}(\omega)\right|\left(\left|f^{\prime}(\omega)\right|^{2}+\Im m f(\omega)\right)^{\alpha / 2-l} \\
& \leq C_{1} \sum_{\alpha=0}^{l} \sup _{\omega \in \mathbb{R}}\left|d^{\alpha} g_{R}(\omega)\right| C_{2}^{\alpha / 2-l} .
\end{aligned}
$$

Next, we consider the limit $R \rightarrow \infty$. Because $\theta \in C_{0}^{\infty}(\mathbb{R} ; \mathbb{R})$ and $g \in \mathcal{S}(\mathbb{R})$ and

$$
d^{\alpha} g_{R}(\omega)=\sum_{\beta=0}^{\alpha} d^{\alpha-\beta} g(\omega)\left(\begin{array}{c}
\alpha \\
\beta
\end{array}\right) \frac{1}{R^{\beta}} d^{\beta} \theta(\omega)
$$

it follows that

$$
\left|d^{\alpha} g_{R}(\omega)-d^{\alpha} g(\omega)\right| \leq\left|\sum_{\beta=1}^{\alpha} d^{\alpha-\beta} g(\omega)\left(\begin{array}{c}
\alpha \\
\beta
\end{array}\right) \frac{1}{R^{\beta}} d^{\beta} \theta(\omega)\right|=\mathcal{O}\left(R^{-1}\right) .
$$

and from (2.14) and the assumption $\tau \geq 0$ it follows

$$
\begin{aligned}
& \left|\int_{\omega=-\infty}^{\infty} e^{\mathrm{i} \tau f(\omega)} g_{R}(\omega) \mathrm{d} \omega-\int_{\omega=-\infty}^{\infty} e^{\mathrm{i} \tau f(\omega)} g(\omega) \mathrm{d} \omega\right| \\
\leq & \int_{\omega=-\infty}^{\infty} e^{-\tau \Im \mathrm{m} f(\omega)}\left|g_{R}(\omega)-g(\omega)\right| \mathrm{d} \omega \leq \int_{\omega=-\infty}^{\infty}\left|g_{R}(\omega)-g(\omega)\right| \mathrm{d} \omega .
\end{aligned}
$$

Using the definition of $g_{R}$ it follows that

$$
\begin{aligned}
\int_{\omega=-\infty}^{\infty}\left|g_{R}(\omega)-g(\omega)\right| \mathrm{d} \omega & \leq \int_{\omega=-\infty}^{\infty}|g(\omega)||1-\theta(\omega / R)| \mathrm{d} \omega \\
& \leq \int_{|\omega| \geq R}|g(\omega)| \mathrm{d} \omega
\end{aligned}
$$

Since $g \in \mathcal{S}(\mathbb{R})$ the last integral tends to 0 for $R \rightarrow \infty$ and thus (A.1) holds even for the functions $\omega \rightarrow f(\omega)=-\overline{\kappa(\omega)}$ and $\omega \rightarrow$ 
$g(\omega)=\psi_{k}(\tau, \omega)$, although they are not satisfying the assumptions of Theorem A.1.

Because (according to Theorem 2.4), for every $\phi \in \mathcal{S}(\mathbb{R}), \nu[\phi] \in \mathcal{S}(\mathbb{R})$, the operator from the following definition is well-defined.

Definition 2.5 The attenuation solution operator $\mathcal{B}[\cdot]: \mathcal{S}^{\prime} \rightarrow \mathcal{S}^{\prime}$ is defined by

$$
\begin{aligned}
\langle\mathcal{B}[\rho], \phi \otimes \psi\rangle_{\mathcal{S}^{\prime}, \mathcal{S}}= & \langle\rho, \nu[\phi] \otimes \psi\rangle_{\mathcal{S}^{\prime}, \mathcal{S}} \\
& \text { for all } \rho \in \mathcal{S}^{\prime} \text { and } \phi \in \mathcal{S}(\mathbb{R}), \psi \in \mathcal{S}\left(\mathbb{R}^{3}\right) .
\end{aligned}
$$

Remark: In a weakly attenuating medium $\kappa(\omega)=\omega+\mathrm{i} k_{\infty}+k_{*}(\omega)$, and therefore, for every $t, \tau \in \mathbb{R}$

$$
\begin{aligned}
k(t, \tau) & :=\mathcal{F}^{-1}\left[e^{\mathrm{i}\left(\cdot+\mathrm{i} \kappa_{\infty}+k_{*}(\cdot)\right) \tau}\right](t) \\
& =e^{-k_{\infty} \tau} \mathcal{F}^{-1}\left[e^{\mathrm{i} k_{*}(\cdot) \tau}\right](t-\tau) \\
& =e^{-k_{\infty} \tau} \mathcal{F}^{-1}\left[1+\left(e^{\mathrm{i} k_{*}(\cdot) \tau}-1\right)\right](t-\tau) \\
& =\sqrt{2 \pi} e^{-k_{\infty} \tau} \delta(t-\tau)+e^{-k_{\infty} \tau} \mathcal{F}^{-1}\left[e^{\mathrm{i} k_{*}(\cdot) \tau}-1\right](t-\tau) .
\end{aligned}
$$

Because there exists a constant $C>0$ such that

$$
\left|e^{\mathrm{i} k_{*}(\omega) \tau}-1\right| \leq C\left|k_{*}(\omega) \tau\right| \text { for all } \omega, \tau \in \mathbb{R},
$$

it follows from Definition 2.2 (stating that $k_{*} \in L^{2}(\mathbb{R} ; \mathbb{C})$ ) and Plancharel's identity that

$$
\begin{aligned}
\omega & \rightarrow e^{\mathrm{i} k_{*}(\omega) \tau}-1 \in L^{2}(\mathbb{R} ; \mathbb{C}) \text { and } \\
t & \rightarrow e^{-k_{\infty} \tau} \mathcal{F}^{-1}\left[e^{\mathrm{i} k_{*}(\cdot) \tau}-1\right](t-\tau) \in L^{2}(\mathbb{R} ; \mathbb{C}) \text { for all } \tau \in \mathbb{R} .
\end{aligned}
$$

Now, assume that $\rho \in C^{0}\left(\mathbb{R} \times \mathbb{R}^{3} ; \mathbb{C}\right) \cap \mathcal{S}^{\prime}$ with support in $[0, \infty) \times \mathbb{R}^{3}$, $\phi \in \mathcal{S}(\mathbb{R})$ and $\psi \in \mathcal{S}\left(\mathbb{R}^{3}\right)$

$$
\begin{aligned}
& \langle\rho, \nu[\phi] \otimes \psi\rangle_{\mathcal{S}^{\prime}, \mathcal{S}} \\
= & \int_{\mathbb{R}^{3} \tau=-\infty} \int_{\tau}^{\infty} \rho(\tau, \mathbf{x}) \overline{\nu[\phi](\tau) \psi(\mathbf{x})} \mathrm{d} \tau \mathrm{d} \mathbf{x} \\
= & \frac{1}{\sqrt{2 \pi}} \int_{\mathbb{R}^{3}} \overline{\psi(\mathbf{x})} \int_{\tau=-\infty}^{\infty} \rho(\tau, \mathbf{x}) \int_{\omega=-\infty}^{\infty} e^{\mathrm{i} \kappa(\omega) \tau} \hat{\bar{\phi}}(\omega) \mathrm{d} \omega \mathrm{d} \tau \mathrm{d} \mathbf{x} .
\end{aligned}
$$


Using Parseval's identity we get

$$
\begin{aligned}
& \langle\rho, \nu[\phi] \otimes \psi\rangle_{\mathcal{S}^{\prime}, \mathcal{S}} \\
= & \frac{1}{\sqrt{2 \pi}} \int_{\mathbb{R}^{3}} \int_{\tau=-\infty}^{\infty} \rho(\tau, \mathbf{x}) \overline{\psi(\mathbf{x})} \int_{t=-\infty}^{\infty} k(t, \tau) \overline{\phi(t)} \mathrm{d} t \mathrm{~d} \tau \mathrm{d} \mathbf{x} \\
= & \int_{\mathbb{R}^{3}} \int_{t=-\infty}^{\infty} \overline{\psi(\mathbf{x}) \phi(t)} \underbrace{\left(\frac{1}{\sqrt{2 \pi}} \int_{\tau=-\infty}^{\infty} k(t, \tau) \rho(\tau, \mathbf{x}) \mathrm{d} \tau\right)}_{=\mathcal{B}[\rho](t, \mathbf{x})} \mathrm{d} t \mathrm{~d} \mathbf{x} .
\end{aligned}
$$

Then for $\rho \in C^{0}\left(\mathbb{R} \times \mathbb{R}^{3} ; \mathbb{C}\right) \cap \mathcal{S}^{\prime}$ it follows from (2.18) and (2.17) that

$$
e^{k_{\infty} t} \mathcal{B}[\rho](t, \mathbf{x})=(\mathcal{I} d+\mathcal{T})[\rho](t, \mathbf{x})
$$

where

$$
\mathcal{T}[\rho](t, \mathbf{x})=\frac{1}{\sqrt{2 \pi}} \int_{\tau=-\infty}^{\infty} e^{k_{\infty}(t-\tau)} \mathcal{F}^{-1}\left[e^{\mathrm{i} k_{*}(\omega) \tau}-1\right](t-\tau) \rho(\tau, \mathbf{x}) \mathrm{d} \tau
$$

Theorem 2.6 Let $q=\mathcal{I}[p]$ and $q^{a}=\mathcal{I}\left[p^{a}\right]$, where $p$ and $p^{a}$ are the solutions of the equations (1.1) and (1.2), respectively. Then

$$
q^{a}=\mathcal{B}[q]
$$

Proof: Let $\phi \in \mathcal{S}(\mathbb{R})$ and $\psi \in \mathcal{S}\left(\mathbb{R}^{3}\right)$. Then, from (2.21), the definition of the Fourier-transform (1.3), the definition of $\mathcal{B}[\cdot]$ in (2.16), because $\nu[\phi] \in \mathcal{S}(\mathbb{R})$ (see (2.12)) and because $q$ solves (2.8) it follows that

$$
\begin{aligned}
& \left\langle\mathcal{A}_{\kappa}[\mathcal{B}[q]], \phi \otimes \psi\right\rangle_{\mathcal{S}^{\prime}, \mathcal{S}}-\left\langle\mathcal{B}[q], \phi \otimes \Delta_{\mathbf{x}} \psi\right\rangle_{\mathcal{S}^{\prime}, \mathcal{S}} \\
= & -\left\langle\mathcal{B}[q] \overline{\kappa^{2} \hat{\phi}} \otimes \psi\right\rangle_{\mathcal{S}^{\prime}, \mathcal{S}}-\left\langle\mathcal{B}[q], \phi \otimes \Delta_{\mathbf{x}} \psi\right\rangle_{\mathcal{S}^{\prime}, \mathcal{S}} \\
= & \left.-\left\langle q, \nu \overline{\overline{\kappa^{2}} \hat{\phi}}\right] \otimes \psi\right\rangle_{\mathcal{S}^{\prime}, \mathcal{S}}-\left\langle q, \nu[\phi] \otimes \Delta_{\mathbf{x}} \psi\right\rangle_{\mathcal{S}^{\prime}, \mathcal{S}} \\
= & \left.-\left\langle q, \nu \overline{\overline{\kappa^{2}} \hat{\phi}}\right] \otimes \psi\right\rangle_{\mathcal{S}^{\prime}, \mathcal{S}}-\left\langle q, \partial_{\tau \tau} \nu[\phi] \otimes \psi\right\rangle_{\mathcal{S}^{\prime}, \mathcal{S}} \\
& +\nu[\phi](0)\langle h, \psi\rangle_{\mathcal{S}^{\prime}\left(\mathbb{R}^{3}\right), \mathcal{S}\left(\mathbb{R}^{3}\right)}
\end{aligned}
$$

We are representing every term on the right hand side: 
1. From Theorem 2.4 it follows that

$$
\nu[\phi](0)=\frac{1}{\sqrt{2 \pi}} \int_{\omega=-\infty}^{\infty} \hat{\phi}(\omega) \mathrm{d} \omega=\check{\hat{\phi}}(0)=\phi(0)
$$

and thus

$$
\nu[\phi](0)\langle h, \psi\rangle_{\mathcal{S}^{\prime}\left(\mathbb{R}^{3}\right), \mathcal{S}\left(\mathbb{R}^{3}\right)}=\phi(0)\langle h, \psi\rangle_{\mathcal{S}^{\prime}\left(\mathbb{R}^{3}\right), \mathcal{S}\left(\mathbb{R}^{3}\right)} .
$$

2. The first term on the right hand side of (2.22) can be represented as follows:

$$
\left\langle q, \nu\left[\overline{\overline{\kappa^{2}} \hat{\phi}}\right] \otimes \psi\right\rangle_{\mathcal{S}^{\prime}, \mathcal{S}}=\left\langle q, \frac{1}{\sqrt{2 \pi}} \int_{\omega=-\infty}^{\infty} \overline{\kappa^{2}(\omega)} e^{-\mathrm{i} \overline{\kappa(\omega)}} \hat{\phi}(\omega) \mathrm{d} \omega \otimes \psi\right\rangle_{\mathcal{S}^{\prime}, \mathcal{S}}
$$

3. For the second term we find that

$$
\begin{aligned}
\left\langle q, \partial_{\tau \tau} \nu[\phi] \otimes \psi\right\rangle_{\mathcal{S}^{\prime}, \mathcal{S}} & =\left\langle q, \frac{1}{\sqrt{2 \pi}} \int_{\omega=-\infty}^{\infty} \partial_{\tau \tau} e^{-\mathrm{i} \overline{\kappa(\omega)}} \cdot \hat{\phi}(\omega) \mathrm{d} \omega \otimes \psi\right\rangle_{\mathcal{S}^{\prime}, \mathcal{S}} \\
& =-\left\langle q, \frac{1}{\sqrt{2 \pi}} \int_{\omega=-\infty}^{\infty} \overline{\kappa^{2}(\omega)} e^{-\mathrm{i} \overline{(\omega)}} \cdot \hat{\phi}(\omega) \mathrm{d} \omega \otimes \psi\right\rangle_{\mathcal{S}^{\prime}, \mathcal{S}} .
\end{aligned}
$$

The sum of the first and second term vanishes and thus from (2.22) it follows that

$$
\begin{aligned}
& \left\langle\mathcal{A}_{\kappa}[\mathcal{B}[q]], \phi \otimes \psi\right\rangle_{\mathcal{S}^{\prime}, \mathcal{S}}-\left\langle\mathcal{B}[q], \phi \otimes \Delta_{\mathbf{x}} \psi\right\rangle_{\mathcal{S}^{\prime}, \mathcal{S}} \\
= & \phi(0)\langle h, \psi\rangle_{\mathcal{S}^{\prime}\left(\mathbb{R}^{3}\right), \mathcal{S}\left(\mathbb{R}^{3}\right)},
\end{aligned}
$$

which shows that $\mathcal{B}[q]$ solves (2.7). Since the solution of this equation is unique it follows that $q^{a}=\mathcal{B}[q]$.

\section{Reconstruction formulas}

In this section we provide explicit reconstruction formulas for the absorption density $h$ (the right hand side of (1.2)) in attenuating media. The basis of these formulas are exact reconstruction formulas in non-attenuating media. 
In the case of non-attenuating media the problem of photoacoustic tomography consists in determining the absorption density $h$ from measurement data of

$$
m(t, \boldsymbol{\xi}):=p(t, \boldsymbol{\xi}) \text { for all } t>0, \boldsymbol{\xi} \in \Gamma,
$$

where $\Gamma$ denotes the measurement surface and $p$ is the solution of (1.1).

Let $\mathcal{W}$ be the operator which maps $h$ to $p$. The most universal (meaning applicable for a series of measurement geometries $\Gamma$ ) formula for $\mathcal{W}^{-1}[\cdot]$ is due to $\mathrm{Xu} \&$ Wang [27]. Several different formulas of such kind were presented and analyzed in [14, 16, 12]. The formula of Xu \& Wang [27] in $\mathbb{R}^{3}$ reads as follows:

$$
h(x)=\frac{2}{\Omega_{0}} \int_{\boldsymbol{\xi} \in \Gamma} \frac{p(|\boldsymbol{\xi}-\mathbf{x}|, \boldsymbol{\xi})-|\boldsymbol{\xi}-\mathbf{x}| \frac{\partial p}{\partial t}(|\boldsymbol{\xi}-\mathbf{x}|, \boldsymbol{\xi})}{|\boldsymbol{\xi}-\mathbf{x}|^{2}}\left(\mathbf{n}_{\boldsymbol{\xi}} \cdot \frac{\boldsymbol{\xi}-\mathbf{x}}{|\boldsymbol{\xi}-\mathbf{x}|}\right) \mathrm{d} s(\Gamma)
$$

where $\Omega_{0}$ is $2 \pi$ for a planar geometry and $4 \pi$ for cylindrical and spherical geometries and $\mathbf{n}_{\boldsymbol{\xi}}$ is the outer normal vector for the measurement surface $\Gamma$.

From Theorem 2.6 we get an explicit reconstruction formula in the case of attenuating media:

Theorem 3.1 Under the assumption that the universal back-projection can be applied in the non-attenuating case, we have

$$
h=\mathcal{W}^{-1}\left[\partial_{t} \mathcal{B}^{-1}\left[(t, \boldsymbol{\xi}) \rightarrow q^{a}(t, \boldsymbol{\xi})\right]\right] .
$$

In the following we study the attenuation operator $\mathcal{B}[\cdot]$ and its inverse in weakly attenuating media.

From (2.19) it follows

$$
h=\mathcal{W}^{-1}\left[\partial_{t}(\mathcal{I} d+\mathcal{T})^{-1}\left[(t, \boldsymbol{\xi}) \rightarrow e^{k_{\infty} t} q^{a}(t, \boldsymbol{\xi})\right]\right] .
$$

In particular, in the case of a constantly attenuating medium the kernel of the integral operator $\mathcal{B}[\cdot]$ simplifies to $\left(k_{*}(\omega)=0\right)$

$$
\frac{1}{\sqrt{2 \pi}} \mathcal{F}^{-1}\left[e^{\mathrm{i} \kappa(\cdot) \tau}\right](t)=\frac{1}{\sqrt{2 \pi}} e^{-k_{\infty} \tau} \mathcal{F}^{-1}\left[e^{\mathrm{i} \cdot \tau}\right](t)=e^{-k_{\infty} \tau} \delta(t-\tau) .
$$

Thus from (2.18) it follows that

$$
\mathcal{B}[q](t, \mathbf{x})=\frac{1}{\sqrt{2 \pi}} e^{-k_{\infty} t} q(t, \mathbf{x})
$$


Thus the operator $\mathcal{B}[\cdot]$ is a multiplication operator and the reconstruction formula (3.3) rewrites to

$$
h=\mathcal{W}^{-1}\left[(t, \boldsymbol{\xi}) \rightarrow \partial_{t}\left(e^{k_{\infty} t} q^{a}(t, \boldsymbol{\xi})\right)\right] .
$$

Using that $q=e^{k_{\infty} t} q^{a}$, we get explicit formulas for the time derivatives of $q$ :

$$
\begin{aligned}
\partial_{t} q(t, \boldsymbol{\xi}) & =e^{k_{\infty} t}\left(k_{\infty} q^{a}(t, \boldsymbol{\xi})+\partial_{t} q^{a}(t, \boldsymbol{\xi})\right) \\
\partial_{t t} q(t, \boldsymbol{\xi}) & =e^{k_{\infty} t}\left(k_{\infty}^{2} q^{a}(t, \boldsymbol{\xi})+2 k_{\infty} \partial_{t} q^{a}(t, \boldsymbol{\xi})+\partial_{t t} q^{a}(t, \boldsymbol{\xi})\right) .
\end{aligned}
$$

Therefore, inserting the representation of the derivatives in (3.1) we get

$$
\begin{aligned}
\tilde{q^{a}}(t, \boldsymbol{\xi}) & =\partial_{t} q^{a}(t, \boldsymbol{\xi})-t \partial_{t t} q^{a}(t, \boldsymbol{\xi}) \\
h(\mathbf{x}) & =\frac{2}{\Omega_{0}} \int_{\boldsymbol{\xi} \in \Gamma} \frac{\tilde{q}^{a}(|\boldsymbol{\xi}-\mathbf{x}|, \boldsymbol{\xi})}{|\boldsymbol{\xi}-\mathbf{x}|^{2}}\left(\mathbf{n}_{\boldsymbol{\xi}} \cdot \frac{\boldsymbol{\xi}-\mathbf{x}}{|\boldsymbol{\xi}-\mathbf{x}|}\right) \mathrm{d} s(\Gamma) .
\end{aligned}
$$

\section{Numerical experiments}

In this section we describe an algorithm for photoacoustic inversion in a weakly attenuating medium, in which case the attenuation coefficient is $\omega \rightarrow \kappa(\omega)=\omega+\mathrm{i} k_{\infty}+k_{*}(\omega)$, with $k_{*} \in L^{2}(\mathbb{R} ; \mathbb{C}) \cap C^{\infty}(\mathbb{R} ; \mathbb{C})$.

The numerical inversions and examples will be performed in $\mathbb{R}^{2}$ for the two-dimensional attenuated wave equation. This is consistent with a distributional solution of $(1.2)$ in $\mathbb{R}^{3}$ where $(x, y, z) \rightarrow h(x, y)$ is considered a distribution in $\mathbb{R}^{3}$, which is independent of the third variable. In this case $p^{a}$ can be considered a two-dimensional distribution (1.2), which solves the two-dimensional attenuated wave equation:

$$
\begin{aligned}
\mathcal{A}_{\kappa}\left[p^{a}\right]\left(t, x_{1}, x_{2}\right)-\Delta p^{a}\left(t, x_{1}, x_{2}\right) & =\delta^{\prime}(t) h\left(x_{1}, x_{2}\right), & & t \in \mathbb{R},\left(x_{1}, x_{2}\right) \in \mathbb{R}^{2}, \\
p^{a}\left(t, x_{1}, x_{2}\right) & =0, & & t<0,\left(x_{1}, x_{2}\right) \in \mathbb{R}^{2} .
\end{aligned}
$$

The two-dimensional universal back-projection formula from [2], which is used below, is given by

$$
h(x)=-\frac{4}{\Omega_{0}} \int_{\boldsymbol{\xi} \in \Gamma} \int_{t=|\boldsymbol{\xi}-\mathbf{x}|}^{\infty}\left(\frac{\left(\partial_{t}\left(t^{-1} p\right)\right)(t, \boldsymbol{\xi})}{\sqrt{t^{2}-|\boldsymbol{\xi}-\mathbf{x}|^{2}}} \mathrm{~d} t\right) \mathbf{n}_{\boldsymbol{\xi}} \cdot(\boldsymbol{\xi}-\mathbf{x}) \mathrm{d} s(\Gamma),
$$


where $\Omega_{0}$ is $2 \pi$ for a line measurement geometry and $4 \pi$ for a circular measurement geometry, $\mathbf{n}_{\boldsymbol{\xi}}$ is the outer normal vector for the curve $\Gamma$.

We assume that the attenuated photoacoustic pressure $p^{a}$ is measured on a set of $N$ points on a measurement curve $\Gamma$ at $N_{T}$ uniformly distributed time points

$$
t_{i}=i \Delta_{T}, i=1, \ldots, N_{T} \text { where } \Delta_{T}=\frac{T}{N_{T}} .
$$

In our experiments $\Gamma$ will either be a circle of radius $R$, where the $N$ measurement points are radially uniformly distributed,

$$
\boldsymbol{\xi}_{j}=R\left(\cos \left(j \Delta_{\xi}\right), \sin \left(j \Delta_{\xi}\right)\right), j=0,1, \ldots, N-1 \text { where } \Delta_{\xi}=\frac{2 \pi}{N}
$$

or on a segment of length $2 l$ of the $x$-axis, in which case

$$
\boldsymbol{\xi}_{j}=\left(2 j \Delta_{x}-1,0\right), \text { where } \Delta_{x}=l / N .
$$

In this case we consider $h$ to be supported in the upper half-space.

The evaluation of the integral operator $\mathcal{I}$ [.] is numerically realized as follows: For every measurement point $\left\{\boldsymbol{\xi}_{j}: j=0,1, \ldots, N-1\right\}$

$$
q^{a}\left(t_{i}, \boldsymbol{\xi}_{j}\right)=\Delta_{T} \sum_{n=1}^{i} p^{a}\left(t_{n}, \boldsymbol{\xi}_{j}\right)
$$

The relation $q^{a}=\mathcal{B}[q]$ from $(2.21)$ is realized numerically as follows: Because we assume a weakly attenuation medium $\mathcal{B}[\cdot]$ (defined in (2.16)) is an integral operator with kernel $k$ defined in (2.17). We use the Taylor-series expansion of the exponential function $\tau \rightarrow e^{\mathrm{i} k_{*}(\omega) \tau}$ and get

$$
\mathcal{F}^{-1}\left[e^{\mathrm{i} k_{*}(\omega) \tau}-1\right](t)=\sum_{k=1}^{\infty} \frac{\tau^{k}}{k !} \mathcal{F}^{-1}\left[\left(\mathrm{i} k_{*}(\omega)\right)^{k}\right](t) .
$$

Inserting (4.4) into (2.20) and taking into account (2.19) and (2.21) we get for all $i=1, \ldots, N_{T}$ that

$$
\begin{aligned}
& q^{a}\left(t_{i}, \boldsymbol{\xi}_{j}\right) \\
= & e^{-k_{\infty} t_{i}} q\left(t_{i}, \boldsymbol{\xi}_{j}\right)+\frac{1}{\sqrt{2 \pi}} \int_{\tau=-\infty}^{\infty} e^{-k_{\infty} \tau} \sum_{k=1}^{\infty} \frac{\tau^{k}}{k !} r_{k}\left(t_{i}-\tau\right) q\left(\tau, \boldsymbol{\xi}_{j}\right) \mathrm{d} \tau,
\end{aligned}
$$

where

$$
s \rightarrow r_{k}(s):=\mathcal{F}^{-1}\left[\mathrm{i}^{k} k_{*}^{k}(\omega)\right](s) .
$$


The integral on the right hand side of (4.5) is approximated for numerical purposes as follows:

$$
\frac{\Delta_{T}}{\sqrt{2 \pi}} \sum_{m=1}^{N_{T}} e^{-k_{\infty} t_{m}} \sum_{k=1}^{\infty} \frac{t_{m}^{k}}{k !} r_{k}\left(t_{i}-t_{m}\right) q\left(t_{m}, \boldsymbol{\xi}_{j}\right)
$$

This expression is represented as a matrix-vector multiplication with vector $\vec{q}_{j}=\left(q\left(t_{m}, \boldsymbol{\xi}_{j}\right)\right)_{m=1, \ldots, N_{T}}$ and matrix with entries

$$
b_{i m}=\frac{\Delta_{T}}{\sqrt{2 \pi}} e^{-k_{\infty} t_{m}} \sum_{k=1}^{\infty} \frac{t_{m}^{k}}{k !} r_{k}\left(t_{i}-t_{m}\right) \text { with } 1 \leq i \leq N_{T}, \text { and } 1 \leq m \leq N_{T} .
$$

Then it follows from (4.5) that

$$
\vec{q}_{j}^{a}=\left(e^{-k_{\infty} t_{i}} I+B\right) \vec{q}_{j},
$$

which is the discretized version of (2.19). To get numerical values for the entries of $B$, the terms $r_{k}\left(t_{i}-t_{m}\right)$ have to be numerically calculated. For $k=1$,

$$
r_{1}(t)=\frac{1}{\sqrt{2 \pi}} \int_{\omega=-\infty}^{\infty} \mathrm{i} k_{*}(\omega) e^{-\mathrm{i} \omega t} \mathrm{~d} \omega,
$$

which can be evaluated by numerical integration for all $t_{i}$. When $k>1, r_{k}$ is a convolution of $r_{k-1}$ and $r_{1}$ and thus

$$
r_{k}(t)=\frac{1}{\sqrt{2 \pi}}\left(r_{1} * r_{k-1}\right)(t)=\frac{1}{\sqrt{2 \pi}} \int_{0}^{t} r_{1}(\tau) r_{k-1}(t-\tau) \mathrm{d} \tau .
$$

Numerically, we approximate the convolution by

$$
r_{k}\left(t_{i}\right) \approx \frac{\Delta_{T}}{\sqrt{2 \pi}} \sum_{m=1}^{i} r_{1}\left(t_{m}\right) r_{k-1}\left(t_{i}-t_{m}\right) .
$$

We summarize the inversion process in a pseudo-code, where we truncate the Taylor-series (4.4) at the tenth coefficient (the number ten has been found from numerical simulations): 
Data: The measurements are denoted by $P_{i, j}^{a}=p^{a}\left(t_{i}, \boldsymbol{\xi}_{j}\right)$ for all $i=1, \ldots, N_{T}$ and $j=0, \ldots, N-1$

Result: Numerical calculation of the absorption density $h_{l}=h\left(x_{l}\right)$

for $1 \leq i \leq N_{T}$ do

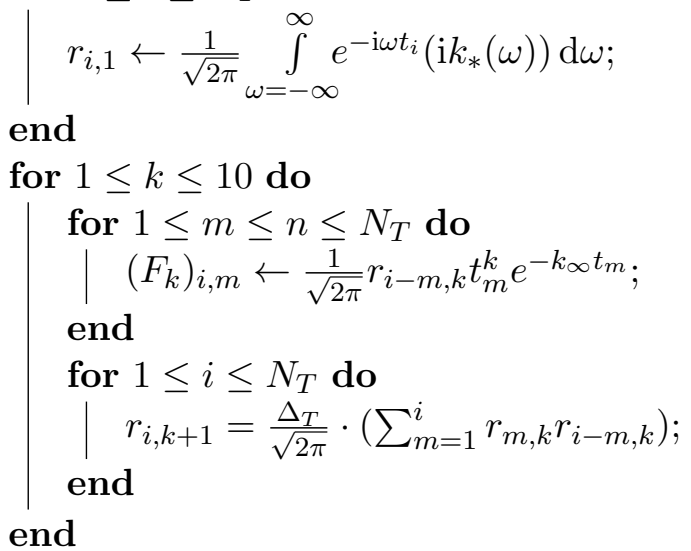

$B \leftarrow \operatorname{diag}\left(e^{-k_{\infty} t_{1}}, e^{-k_{\infty} t_{2}}, \ldots\right)+\Delta_{T} \sum_{k=1}^{10} \frac{F_{k}}{k !} ;$

for $1 \leq i \leq N_{T}$ do

$Q_{i, j}^{a} \leftarrow Q_{i-1, j}^{a}+\Delta_{T} P_{i, j}^{a} ;$

end

$Q \leftarrow Q^{a} B^{-1}$

for $1 \leq i \leq N_{T}$ do

$P_{i, j} \leftarrow \frac{Q_{i, j}-Q_{i-1, j}}{\Delta_{T}} ;$

end

Calculate $h_{l}$ by applying the back-projection operator $\mathcal{W}^{-1}[\cdot]$ on $P_{i, j}$;

Algorithm 1: Pseudocode for reconstructing the absorption density $h$.

\section{Numerical experiments}

We assume that $h$ is a function with compact support in $\mathbb{R}^{2}$. We calculated $p$, the solution of (1.1) using the k-wave toolbox [23]. By integrating $p$ at the points $\boldsymbol{\xi}_{j}, j=0, \ldots, N-1$ over time with $(4.3)$ we get $q\left(t_{i}, \boldsymbol{\xi}_{j}\right)$ for $i=1, \ldots, N_{T}$ and $j=0, \ldots, N-1$. Then we find $q^{a}\left(t_{i}, \boldsymbol{\xi}_{j}\right)$ by matrix-vector multiplication (4.8).

In order to avoid inverse crimes we used different discretization points in space and time for the simulation of the forward data and the inversion. The forward problem is simulated with $N_{T}=500$ and $N=896$, while the inverse problem is solved with $N_{T}=443$ and $N=849$. The absorption density function $h: \mathbb{R}^{2} \rightarrow \mathbb{R}$ with support in $(-0.8,0.8)^{2}$ is the SheppLogan phantom [19]. In all numerical experiments the material parameter 
$k_{\infty}=0.45$

\section{Circular measurement geometry}

In these examples the measurement geometry is a circle with radius $R=$ 1.7 on which there are recorded data on $N=849$ uniformly distributed measurement points. Moreover, the time length is 6 and thus $\Delta_{T}=6 / N_{T}=$ $6 / 443$.

We consider a constantly attenuating medium, with attenuation coefficient $\kappa(\omega)=\omega+\mathrm{i} k_{\infty}$. Figure 2 shows the ground truth (top left) and the simulated pressure data $p^{a}$ on $\Gamma$ over time. Two reconstructions are presented: The first one is obtained by applying the universal back-projection formula (4.2) (middle left), while the middle right image shows the reconstruction obtained with Algorithm 1. The quantitative values of ground truth and the two reconstructions are plotted on the bottom.

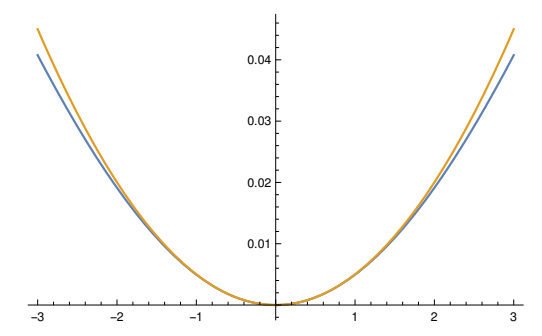

Figure 1: Blue curve corresponds to $\Im m \kappa(\omega)$ of NSW model. Red curve corresponds to power law $0.005 \omega^{2}$.

Next we consider the Nachman, Smith and Waag (NSW) [15] attenuation model:

$$
\kappa(\omega)=\omega \sqrt{\frac{1-\mathrm{i} \omega \tilde{\tau}}{1-\mathrm{i} \omega \tau}}=\omega+\frac{\tau-\tilde{\tau}}{2 \tau \tilde{\tau}} \mathrm{i}+k_{*}(\omega)
$$

where $k_{*}(\omega)=\mathcal{O}\left(|\omega|^{-1}\right)$. Therefore, $\kappa$ is a weak attenuation coefficient with $k_{\infty}=\frac{\tau-\tilde{\tau}}{2 \tau \tilde{\tau}}$. In Figure 3 we present ground truth, simulated measurements $p^{a}$, and compare three imaging techniques:

- Applying the universal back-projection formula $\mathcal{W}^{-1}\left[p^{a}\right]$ (4.2) (thus neglecting the attenuation).

- The compensated back-projection formula

$$
\mathcal{W}^{-1}\left[(t, \boldsymbol{\xi}) \mapsto \partial_{t}\left(e^{k_{\infty} t} q^{a}(t, \boldsymbol{\xi})\right)\right],
$$

which neglects $k_{*}(\omega)$ but takes into account $k_{\infty}$. 
- Reconstruction using (3.2) with the numerical code described in Algorithm 1.

The parameters of attenuation coefficient in the NSW model are $\tilde{\tau}=0.1$ and $\tau=0.11$. For small frequencies the NSW coefficients behaves like a power law of order 2. However, asymptotically, for large frequencies, it behaves like $\omega+\frac{\tau-\tilde{\tau}}{2 \tau \tilde{\tau}}$ i. The NSW-coefficient has been plotted in Figure 1. In order to demonstrate the stability of the algorithm, we also performed reconstructions from noisy data, where a uniformly distributed noise is added with a variance of $20 \%$ of the maximal intensity. The reconstruction results are depicted in the last image of Figure 2 and Figure 3, respectively. 

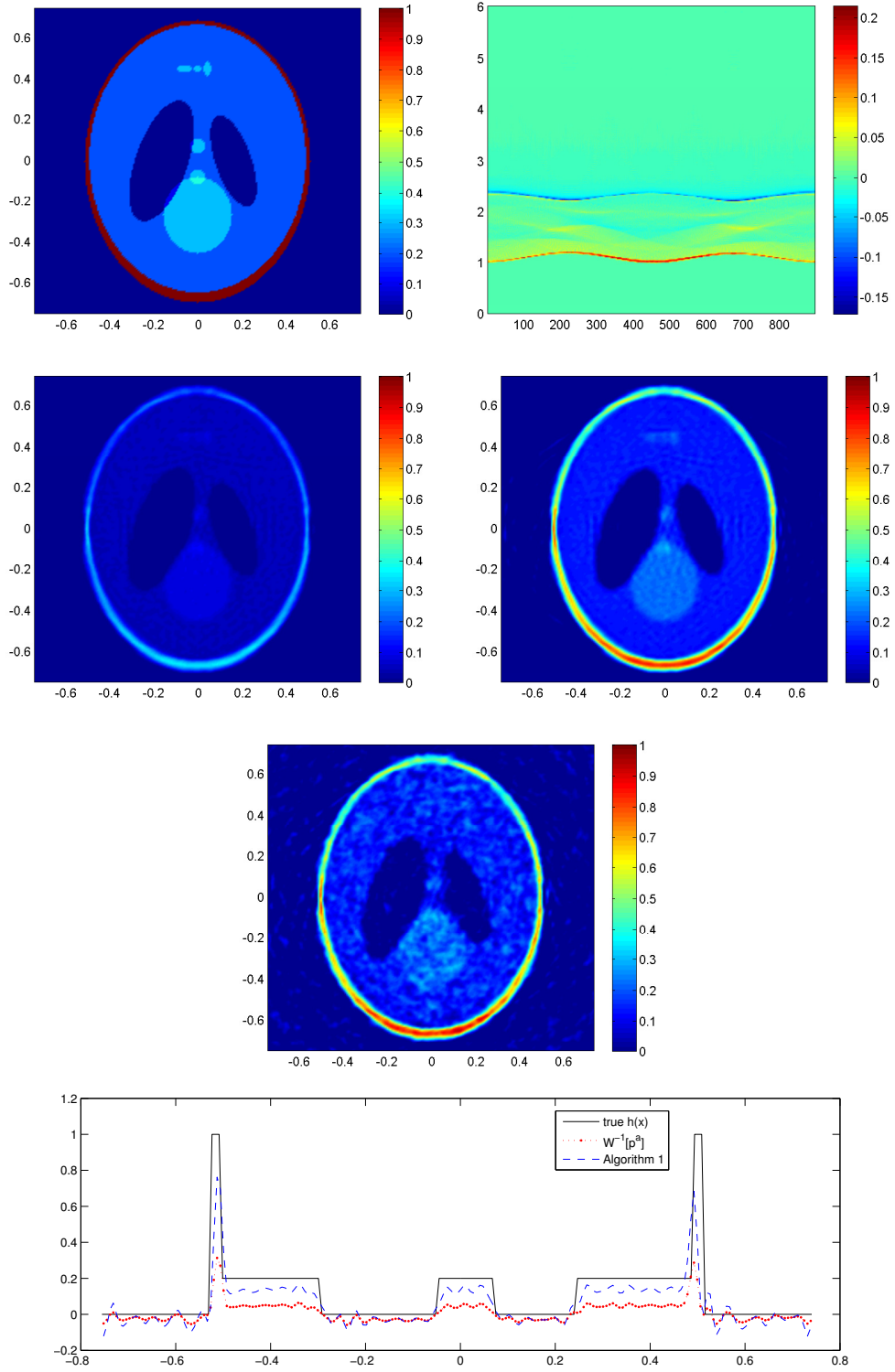

Figure 2: Measurements along a circle and constantly attenuating model. Top left: Ground truth. Top Right: the simulated pressure data $p^{a}$. Middle: Reconstruction by universal back-projection (not taking into account attenuation), and by Algorithm 1 with noise-free and 20\% noise. Bottom: Cross section through ground truth and reconstructions. 

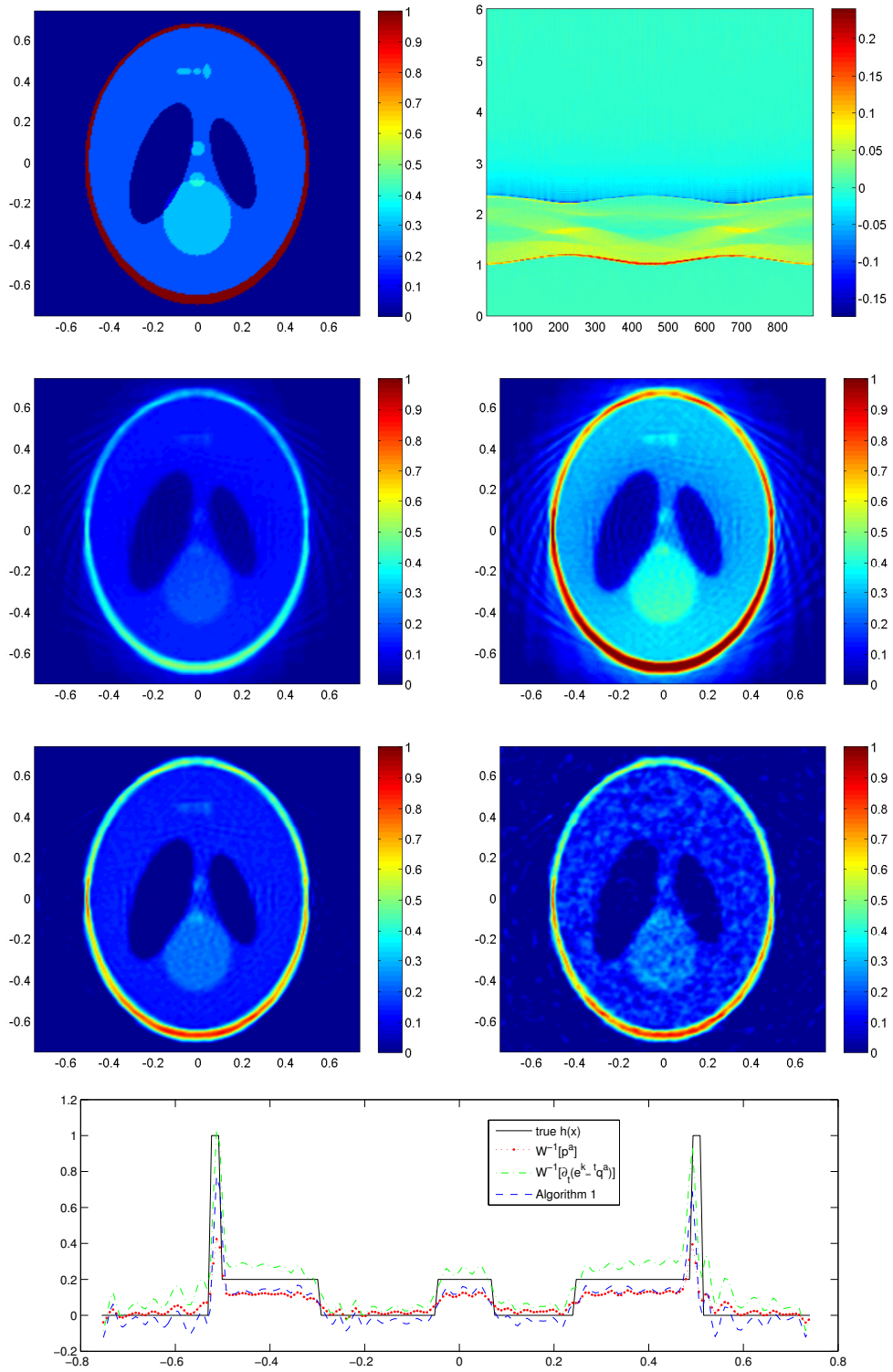

Figure 3: Measurements along a circle and NSW model. Top left: Ground truth. Top Right: the simulated pressure data $p^{a}$. Middle: Reconstruction by universal back-projection (not taking into account attenuation), by compensated attenuation (4.12) and by Algorithm 1 with noise-free and 20\% noise. Bottom: Cross section through ground truth and reconstructions. 


\section{Measurements on a line}

The measurement points are $N=849$ uniformly distributed on a line segment with length $l=10.2$. The distance of the line to the center of the phantom is 1.7. The time length is 8 and thus $\Delta_{T}=8 / 443$.

We consider a constantly attenuating medium, with attenuation coefficient $\kappa(\omega)=\omega+\mathrm{i} k_{\infty}$. Figure 4 shows the ground truth (top left) and the simulated pressure data $p^{a}$ on $\Gamma$ over time. Two reconstructions are presented: The first one is obtained by applying the universal back-projection formula (4.2) (middle left) and the middle right image shows the reconstruction obtained with Algorithm 1. The quantitative values of ground truth and the two reconstructions are plotted on the bottom.

In Figure 5 we present ground truth, simulated measurements $p^{a}$ using NSW model, and compare three imaging techniques, the universal backprojection formula neglecting attenuation, the compensated back-projection formula (4.12), which neglects $k_{*}(\omega)$ but takes into account $k_{\infty}$, and reconstruction with Algorithm 1. The parameters of the NSW attenuation coefficient are again $\tilde{\tau}=0.1$ and $\tau=0.11$. The reconstruction results from noisy data are depicted in the last image of Figure 4 and Figure 5, where uniformly distributed noise is added with a variance of $20 \%$ of the maximal intensity value. Numerical results show that the algorithm is quite stable even with $20 \%$ noise. 

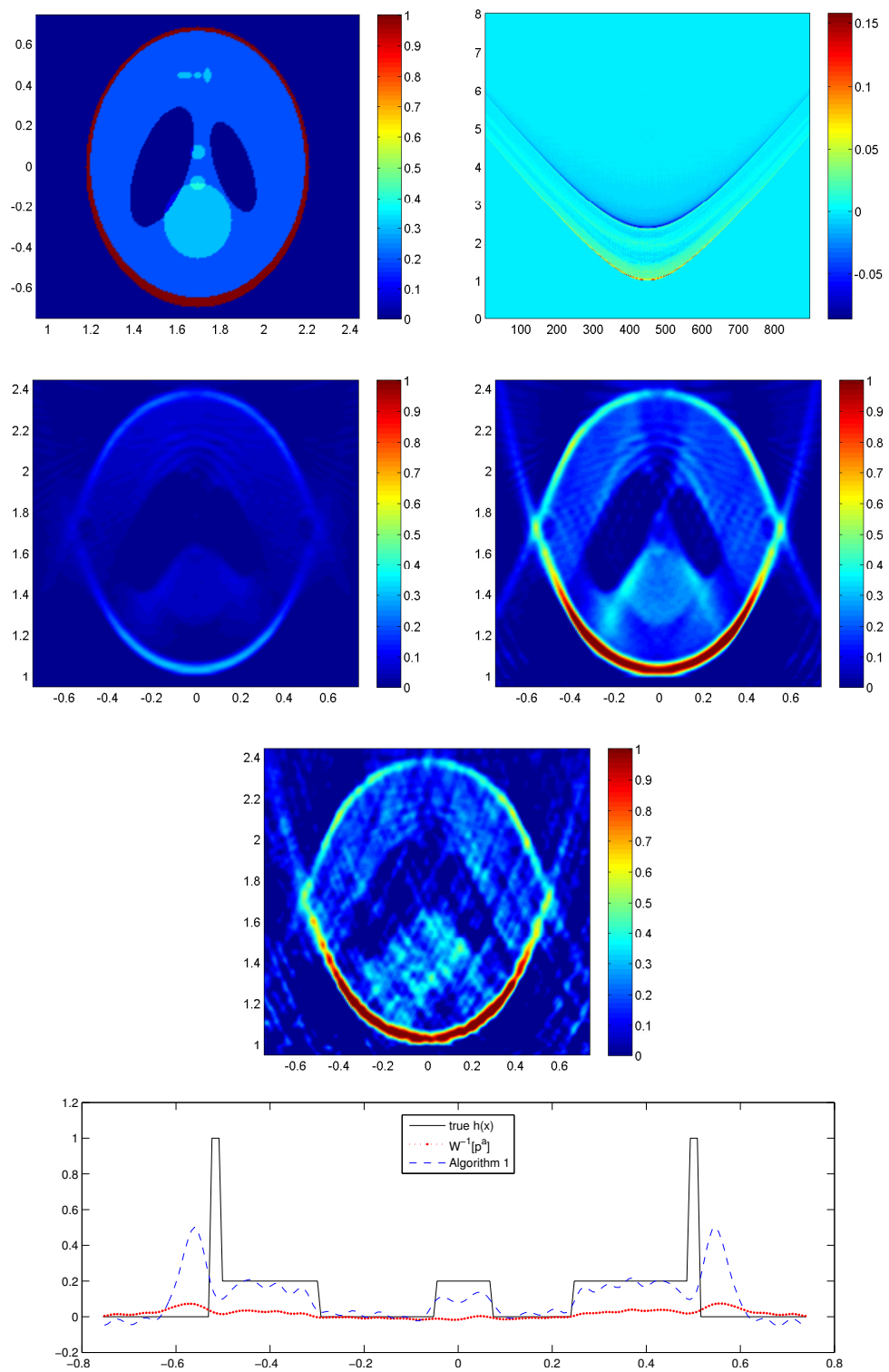

Figure 4: Measurements along a line and constantly attenuating model. Top left: Ground truth. Top Right: the simulated pressure data $p^{a}$. Middle: Reconstruction by universal back-projection (not taking into account attenuation), and by Algorithm 1 with noise-free and 20\% noise. Bottom: Cross section through ground truth and reconstructions. 

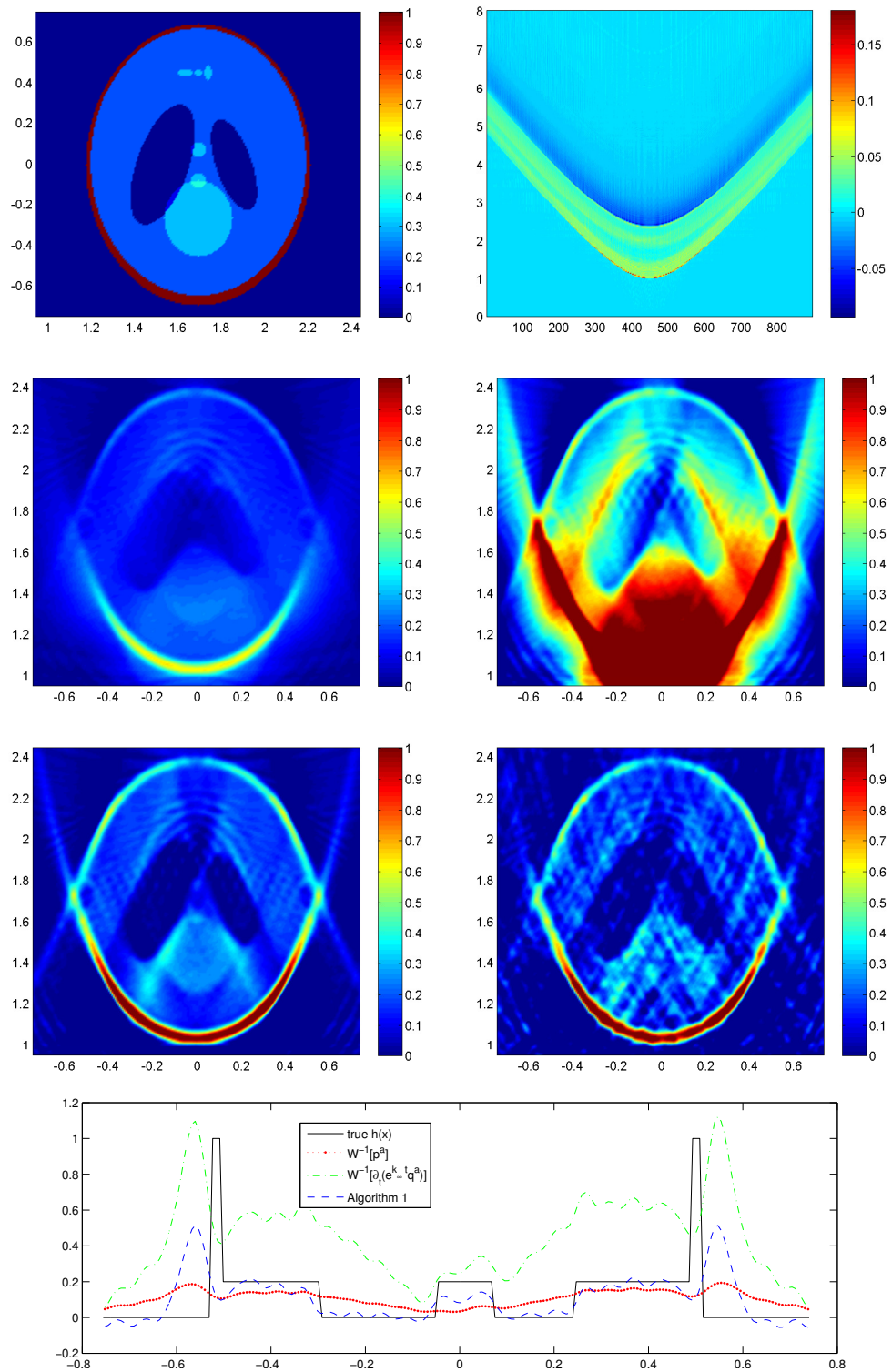

Figure 5: Measurements along a line and NSW model. Top left: Ground truth. Top Right: the simulated pressure data $p^{a}$. Middle: Reconstruction by universal back-projection (not taking into account attenuation), by compensated attenuation (4.12) and by Algorithm 1 with noise-free and 20\% noise. Bottom: Cross section through ground truth and reconstructions. 


\section{Conclusion}

We have presented explicit reconstruction formulas for photoacoustic imaging in acoustically attenuating media, which are based on the universal backprojection formula. We have presented a numerical algorithm and showed numerical reconstructions, which were compared with attenuation compensation techniques. The numerical simulations show that the new technique can produce better visualization in $2 \mathrm{D}$ with a similar numerical complexity.

\section{Acknowledgment}

The work is support by the "Doctoral Program Dissipation and Dispersion in Nonlinear PDEs" (W1245). OS is also supported by the FWF-project "Interdisciplinary Coupled Physics Imaging" (FWF P26687).

\section{References}

[1] H. Ammari, E. Bretin, J. Garnier, and A. Wahab. "Noise source localization in an attenuating medium". In: SIAM Journal on Applied Mathematics 72.1 (2012), pp. 317-336. ISSN: 0036-1399. DOI: 10.1137/ 11083191X. URL: http://dx.doi.org/10.1137/11083191X (cit. on p. 2).

[2] P. Burgholzer, H. Grün, M. Haltmeier, R. Nuster, and G. Paltauf. "Compensation of acoustic attenuation for high-resolution photoacoustic imaging with line detectors". In: Photons Plus Ultrasound: Imaging and Sensing 2007: The Eighth Conference on Biomedical Thermoacoustics, Optoacoustics, and Acousto-optics. Ed. by A. A. Oraevsky and L. V. Wang. Vol. 6437. Proceedings of SPIE. San Jose, CA, USA: SPIE, 2007, p. 643724. DOI: 10.1117/12.700723. URL: http://dx . doi.org/10.1117/12.700723 (cit. on pp. 2, 14).

[3] B. T. Cox and B. E. Treeby. "Artifact trapping during time reversal photoacoustic imaging for acoustically heterogeneous media". In: IEEE Transactions on Medical Imaging 29.2 (2010), pp. 387-396. ISSN: 0278-0062 (cit. on p. 2).

[4] P. Elbau, O. Scherzer, and C. Shi. Singular Values of the Attenuated Photoacoustic Imaging Operator. Preprint on ArXiv arXiv:1611.05807. University of Vienna, Austria, 2016. URL: https://arxiv.org/abs/ 1611.05807 (cit. on pp. 2, 4-6, 28). 
[5] A. Hanyga and M. Seredyńska. "Power-law attenuation in acoustic and isotropic anelastic media". In: Geophysical Journal International 155 (2003), pp. 830-838 (cit. on p. 2).

[6] L. Hörmander. The Analysis of Linear Partial Differential Operators I. 2nd ed. New York: Springer Verlag, 2003 (cit. on p. 28).

[7] C. Huang, L. Nie, R. W. Schoonover, L.V. Wang, and M. A. Anastasio. "Photoacoustic computed tomography correcting for heterogeneity and attenuation". In: Journal of Biomedical Optics 17 (2012), p. 061211. ISSN: 1083-3668 (cit. on p. 2).

[8] K. Kalimeris and O. Scherzer. "Photoacoustic imaging in attenuating acoustic media based on strongly causal models". In: Math. Methods Appl. Sci. 36.16 (Feb. 2013), pp. 2254-2264. ISSN: 1099-1476. DOI: 10.1002/mma. 2756. URL: http://dx . doi .org/10 .1002/mma. 2756 (cit. on p. 2).

[9] L. E. Kinsler, A. R. Frey, A. B. Coppens, and J. V. Sanders. Fundamentals of Acoustics. 4th ed. New York: Wiley, 2000. ISBN: 0-47184789-5 (cit. on p. 2).

[10] R. Kowar and O. Scherzer. "Attenuation Models in Photoacoustics". In: Mathematical Modeling in Biomedical Imaging II: Optical, Ultrasound, and Opto-Acoustic Tomographies. Ed. by H. Ammari. Vol. 2035. Lecture Notes in Mathematics. Berlin Heidelberg: Springer Verlag, 2012 , pp. 85-130. ISBN: 978-3-642-22989-3. DOI: $10.1007 / 978-3-642-$ 22990-9. URL: http://dx.doi.org/10.1007/978-3-642-22990-9 (cit. on p. 2).

[11] R. Kowar, O. Scherzer, and X. Bonnefond. "Causality analysis of frequency-dependent wave attenuation". In: Math. Methods Appl. Sci. 34 (1 2011), pp. 108-124. ISSN: 1099-1476 (online). DOI: 10.1002/mma. 1344. URL: http://dx.doi.org/10.1002/mma.1344 (cit. on p. 2).

[12] P. Kuchment. "Mathematics of hybrid imaging: a brief review". In: The Mathematical Legacy of Leon Ehrenpreis. Ed. by I. Sabadini and D. C. Struppa. Berlin: Springer, 2012, pp. 183-208 (cit. on p. 13).

[13] P. Kuchment. The Radon Transform and Medical Imaging. Philadelphia: Society for Industrial and Applied Mathematics, 2014. ISBN: 9781-611973-28-0 (cit. on p. 2).

[14] P. Kuchment and L. Kunyansky. "Mathematics of thermoacoustic tomography". In: European Journal of Applied Mathematics 19 (2008), pp. 191-224. DOI: 10.1017/S0956792508007353 (cit. on pp. 2, 13). 
[15] A. I. Nachman, J. F. Smith III, and R. C. Waag. "An equation for acoustic propagation in inhomogeneous media with relaxation losses". In: J. Acoust. Soc. Amer. 88.3 (1990), pp. 1584-1595 (cit. on pp. 2, 18).

[16] F. Natterer. "Photo-acoustic inversion in convex domains". In: Inverse Problems and Imaging 6.2 (2012), pp. 1-6. ISSN: 1930-8337 (cit. on p. 13).

[17] H. M. Nussenzveig. Causality and dispersion relations. New York: Academic Press, 1972 (cit. on p. 28).

[18] R. T. Seeley. "Extension of $C^{\infty}$ functions defined in a half space". In: Proceedings of the American Mathematical Society 15 (1964), pp. 625626. ISSN: 0002-9939 (cit. on p. 8).

[19] L. A. Shepp and Y. Vardi. "A statistical model for positron emission tomography". In: IEEE Transactions on Nuclear Science NS-21 (1974), pp. 21-43 (cit. on p. 17).

[20] N. V. Sushilov and R. S. C. Cobbold. "Frequency-domain wave equation and its time-domain solution in attenuating media". In: Journal of the Acoustical Society of America 115 (2005), pp. 1431-1436 (cit. on p. 2).

[21] T.L. Szabo. "Causal theories and data for acoustic attenuation obeying a frequency power law". In: Journal of the Acoustical Society of America 97 (1995), pp. 14-24 (cit. on p. 2).

[22] T.L. Szabo. "Time domain wave equations for lossy media obeying a frequency power law". In: Journal of the Acoustical Society of America 96 (1994), pp. 491-500 (cit. on p. 2).

[23] B. E. Treeby and B. T. Cox. "K-Wave: MATLAB toolbox for the simulation and reconstruction of photoacoustic wace fields". In: Journal of Biomedical Optics 15 (2 2010), p. 021314. ISSN: 1083-3668 (cit. on p. 17).

[24] B. E. Treeby, E. Z. Zhang, and B. T. Cox. "Photoacoustic tomography in absorbing acoustic media using time reversal". In: Inverse Problems 26 (11 2010), p. 115003 (cit. on p. 2).

[25] K. Wang and M. A. Anastasio. "Photoacoustic and thermoacoustic tomography: image formation principles". In: Handbook of Mathematical Methods in Imaging. Ed. by O. Scherzer. Springer, 2011, pp. 781-817 (cit. on p. 2). 
[26] L. V. Wang, ed. Photoacoustic Imaging and Spectroscopy. Optical Science and Engineering. Boca Raton: CRC Press, 2009. xii+499 (cit. on p. 1).

[27] M. Xu and L. V. Wang. "Universal back-projection algorithm for photoacoustic computed tomography". In: Physical Review E 71.1, 016706 (2005). DOI: 10.1103/PhysRevE.71.016706 (cit. on p. 13).

\section{A Appendix}

Theorem A.1 [6, Section 7.7] Let $f, g \in C^{\infty}(\mathbb{R} ; \mathbb{C})$ satisfying

- $\operatorname{supp} g$ is compact and

- $\Im m f(\omega) \geq 0$.

Then there exists a constant $C_{1}>0$ such that for all $l \in \mathbb{N}$ and all $\tau \geq 0$,

$$
\begin{aligned}
& \tau^{l}\left|\int_{\omega=-\infty}^{\infty} e^{\mathrm{i} \tau f(\omega)} g(\omega) \mathrm{d} \omega\right| \\
& \leq C_{1} \sum_{\alpha=0}^{l} \sup _{\omega \in \mathbb{R}}\left|d^{\alpha} g(\omega)\right|\left(\left|f^{\prime}(\omega)\right|^{2}+\Im \mathrm{m} f(\omega)\right)^{\alpha / 2-l} .
\end{aligned}
$$

Lemma A.2 Let $\kappa$ be an attenuation coefficient (cf. Definition 2.1), then there exists a constant $C_{2}>0$ such that

$$
\left|\kappa^{\prime}(\omega)\right|^{2}+\Im \mathrm{m} \kappa(\omega) \geq C_{2} .
$$

Proof: The fourth assumption of Definition 2.1 ensures that the maximal speed of propagation $c$ is finite. Then from [4, Proposition 2.9], it follows that the holomorphic extension $\tilde{\kappa}$ (cf. Definition 2.1)of $\kappa$ to the upper half plane can be represented as

$$
\tilde{\kappa}(z)=A z+B+\int_{\nu=-\infty}^{\infty} \frac{1+z \nu}{\nu-z} \mathrm{~d} \sigma(\nu), \quad z \in \mathbb{H},
$$

where $A=\frac{1}{c}>0, B \in \mathbb{R}$ are constants, and $\sigma: \mathbb{R} \rightarrow \mathbb{R}$ is a monotonically increasing function of bounded variation.

From [17, Formula (C9)] we know that if $\tilde{\kappa}$ satisfies (A.3), then

$$
\nu \rightarrow \Im \mathrm{m} \kappa(\nu)=\pi\left(1+\nu^{2}\right) \sigma^{\prime}(\nu) \text { for all } \nu \in \mathbb{R} .
$$


Because, by assumption $\kappa \in C^{\infty}(\mathbb{R} ; \mathbb{C})$, and because $\sigma: \mathbb{R} \rightarrow \mathbb{R}$ is a monotonically increasing function of bounded variation, we conclude from (A.4) that $\sigma^{\prime} \in C^{\infty}(\mathbb{R} ; \mathbb{R}) \cap L^{1}(\mathbb{R} ; \mathbb{R})$. Moreover, for all fixed $z \in \mathbb{H}$, because $\nu-z \neq 0$ for all $\nu \in \mathbb{R}$, we have $\nu \rightarrow \frac{1+\nu^{2}}{(\nu-z)^{2}}$ is uniformly bounded and thus the function

$$
\nu \rightarrow \frac{1+\nu^{2}}{(\nu-z)^{2}} \sigma^{\prime}(\nu) \in L^{1}(\mathbb{R} ; \mathbb{C}) .
$$

Differentiation of (A.3) with respect to $z$ and taking into account that $\sigma^{\prime} \in$ $C^{\infty}(\mathbb{R} ; \mathbb{R}) \cap L^{1}(\mathbb{R} ; \mathbb{R})$ yields

$$
\tilde{\kappa}^{\prime}(z)=A+\int_{\nu=-\infty}^{\infty} \frac{\left(1+\nu^{2}\right)}{(\nu-z)^{2}} \sigma^{\prime}(\nu) \mathrm{d} \nu, \quad z \in \mathbb{H} .
$$

Let now $z=\omega+\mathrm{i} \eta$ and take real parts in the above formula to get

$$
\Re \mathrm{e} \tilde{\kappa}^{\prime}(\omega+\mathrm{i} \eta)=A+\int_{\nu=-\infty}^{\infty}\left(1+\nu^{2}\right) \frac{(\nu-\omega)^{2}-\eta^{2}}{\left((\nu-\omega)^{2}+\eta^{2}\right)^{2}} \sigma^{\prime}(\nu) \mathrm{d} \nu .
$$

We are proving now that there exists a constant $C_{r}>0$ such that

$$
\Re \mathrm{e} \kappa^{\prime}(\omega)=\lim _{\eta \rightarrow 0^{+}} \Re \mathrm{e} \tilde{\kappa}^{\prime}(\omega+\mathrm{i} \eta) \geq A-2\left(1+C_{r}\right) \sqrt{\left(1+\omega^{2}\right) \sigma^{\prime}(\omega)} .
$$

Let

$$
\begin{aligned}
& \mathcal{A}_{+}:=\left\{\nu \in \mathbb{R}:|\nu-\omega|^{2} \geq\left(1+\omega^{2}\right) \sigma^{\prime}(\omega)\right\}, \\
& \mathcal{A}_{-}:=\left\{\nu \in \mathbb{R}:|\nu-\omega|^{2}<\left(1+\omega^{2}\right) \sigma^{\prime}(\omega)\right\}, \\
& \mathcal{A}_{0}:=\left\{\hat{\nu} \in \mathbb{R}:|\hat{\nu}|^{2}<\left(1+\omega^{2}\right) \sigma^{\prime}(\omega)\right\} .
\end{aligned}
$$

For $\omega \in \mathbb{R}$ and $\eta>0$ let

$$
\nu \rightarrow \rho_{\omega, \eta}(\nu):=\left(1+\nu^{2}\right) \frac{(\nu-\omega)^{2}-\eta^{2}}{\left((\nu-\omega)^{2}+\eta^{2}\right)^{2}} \sigma^{\prime}(\nu) \text { for all } \omega \neq \nu \in \mathbb{R} .
$$

The function $\rho_{\omega, \eta}$ can be estimated as follows:

$$
\rho_{\omega, \eta}(\nu)=\frac{1+\nu^{2}}{(\nu-\omega)^{2}+\eta^{2}} \underbrace{\frac{(\nu-\omega)^{2}-\eta^{2}}{(\nu-\omega)^{2}+\eta^{2}}}_{\leq 1} \sigma^{\prime}(\nu)
$$

Moreover, since $\nu \in \mathcal{A}_{+}$, we find that

$$
\frac{1+\nu^{2}}{(\nu-\omega)^{2}+\eta^{2}} \leq \frac{1+\nu^{2}}{(\nu-\omega)^{2}}=\underbrace{\frac{1+\nu^{2}}{1+(\nu-\omega)^{2}}}_{\leq 2\left(1+\omega^{2}\right)} \underbrace{\frac{1+(\nu-\omega)^{2}}{(\nu-\omega)^{2}}}_{\leq 1+\left(\left(1+\omega^{2}\right) \sigma^{\prime}(\omega)\right)^{-1}},
$$


where the inequality $\frac{1+\nu^{2}}{1+(\nu-\omega)^{2}} \leq 2\left(1+\omega^{2}\right)$ is a consequence of the algebraic identity $2\left(1+\omega^{2}\right)\left(1+(\nu-\omega)^{2}\right)-\left(1+\nu^{2}\right)=2 \omega^{2}(\nu-\omega)^{2}+(\nu-2 \omega)^{2}+1>0$. Therefore with $C_{\omega}=2\left(1+\omega^{2}\right)\left(1+\left(\left(1+\omega^{2}\right) \sigma^{\prime}(\omega)\right)^{-1}\right)$ it follows that

$$
\left|\rho_{\omega, \eta}(\nu)\right| \leq C_{\omega} \sigma^{\prime}(\nu)
$$

and because $\sigma^{\prime} \in L^{1}(\mathbb{R} ; \mathbb{R})$ the latter means that the functions $\left\{\rho_{\omega, \eta}: \eta>0\right\}$ are uniformly dominated by an $L^{1}(\mathbb{R} ; \mathbb{R})$ function. Therefore we can apply the dominated convergence theorem and get

$$
\lim _{\eta \rightarrow 0^{+}} \int_{\mathcal{A}_{+}} \rho_{\omega, \eta}(\nu) \mathrm{d} \nu=\int_{\mathcal{A}_{+}} \rho_{\omega, 0}(\nu) \mathrm{d} \nu \geq 0 .
$$

To estimate $\int_{\mathcal{A}_{-}} \rho_{\omega, \eta}(\nu) \mathrm{d} \nu$, we use the Taylor's expansion of $\omega \rightarrow(1+$ $\left.(\hat{\nu}+\omega)^{2}\right) \sigma^{\prime}(\hat{\nu}+\omega)$ and get

$$
\left(1+(\hat{\nu}+\omega)^{2}\right) \sigma^{\prime}(\hat{\nu}+\omega)=\left(1+\omega^{2}\right) \sigma^{\prime}(\omega)+\hat{\nu}\left(\left(1+\omega^{2}\right) \sigma^{\prime \prime}(\omega)+2 \omega \sigma^{\prime}(\omega)\right)+r(\hat{\nu}),
$$

with

$$
|r(\hat{\nu})| \leq C_{r} \hat{\nu}^{2} \text { for all } \hat{\nu} \in \mathcal{A}_{-} .
$$

Using the substitution $\nu \rightarrow \hat{\nu}:=\nu-\omega$ and (A.8) we get

$$
\begin{aligned}
& \lim _{\eta \rightarrow 0^{+}} \int_{\mathcal{A}_{-}} \rho_{\omega, \eta}(\nu) \mathrm{d} \nu \\
& =\lim _{\eta \rightarrow 0^{+}} \int_{\mathcal{A}_{0}}\left(1+(\hat{\nu}+\omega)^{2}\right) \sigma^{\prime}(\hat{\nu}+\omega) \frac{\hat{\nu}^{2}-\eta^{2}}{\left(\hat{\nu}^{2}+\eta^{2}\right)^{2}} \mathrm{~d} \hat{\nu} \\
& =\left(1+\omega^{2}\right) \sigma^{\prime}(\omega) \lim _{\eta \rightarrow 0^{+}} \int_{\mathcal{A}_{0}} \frac{\hat{\nu}^{2}-\eta^{2}}{\left(\hat{\nu}^{2}+\eta^{2}\right)^{2}} \mathrm{~d} \hat{\nu} \\
& \quad+\left(\left(1+\omega^{2}\right) \sigma^{\prime \prime}(\omega)+2 \omega \sigma^{\prime}(\omega)\right) \lim _{\eta \rightarrow 0^{+}} \int_{\mathcal{A}_{0}} \hat{\nu} \frac{\hat{\nu}^{2}-\eta^{2}}{\left(\hat{\nu}^{2}+\eta^{2}\right)^{2}} \mathrm{~d} \hat{\nu} \\
& \quad+\lim _{\eta \rightarrow 0^{+}} \int_{\mathcal{A}_{0}} r(\hat{\nu}) \frac{\hat{\nu}^{2}-\eta^{2}}{\left(\hat{\nu}^{2}+\eta^{2}\right)^{2}} \mathrm{~d} \hat{\nu}
\end{aligned}
$$

Plugging in the integral formulas

$$
\int_{-a}^{a} \frac{\nu^{2}-\eta^{2}}{\left(\nu^{2}+\eta^{2}\right)^{2}} \mathrm{~d} \nu=\frac{-2 a}{a^{2}+\eta^{2}} \text { and } \int_{-a}^{a} \nu \frac{\nu^{2}-\eta^{2}}{\left(\nu^{2}+\eta^{2}\right)^{2}} \mathrm{~d} \nu=0
$$


into (A.9) we get for the first term with $a=\sqrt{\left(1+\omega^{2}\right) \sigma^{\prime}(\omega)}$

$$
\begin{aligned}
& \left(1+\omega^{2}\right) \sigma^{\prime}(\omega) \lim _{\eta \rightarrow 0^{+}} \int_{\mathcal{A}_{0}} \frac{\hat{\nu}^{2}-\eta^{2}}{\left(\hat{\nu}^{2}+\eta^{2}\right)^{2}} \mathrm{~d} \hat{\nu} \\
& =\left(1+\omega^{2}\right) \sigma^{\prime}(\omega) \lim _{\eta \rightarrow 0^{+}} \frac{-2 \sqrt{\left(1+\omega^{2}\right) \sigma^{\prime}(\omega)}}{\left(1+\omega^{2}\right) \sigma^{\prime}(\omega)+\eta^{2}}=-2 \sqrt{\left(1+\omega^{2}\right) \sigma^{\prime}(\omega)},
\end{aligned}
$$

and the second integral term in (A.9) is vanishing, and for the third term we get

$$
\left|\int_{\mathcal{A}_{0}} r(\hat{\nu}) \frac{\hat{\nu}^{2}-\eta^{2}}{\left(\hat{\nu}^{2}+\eta^{2}\right)^{2}} \mathrm{~d} \hat{\nu}\right| \leq C_{r} \int_{\mathcal{A}_{0}} 1 \mathrm{~d} \hat{\nu} \leq 2 C_{r} \sqrt{\left(1+\omega^{2}\right) \sigma^{\prime}(\omega)} .
$$

Using the estimates (A.10) and (A.11) in (A.9) we get

$$
\lim _{\eta \rightarrow 0^{+}} \int_{\mathcal{A}_{-}} \rho_{\omega, \eta}(\nu) \mathrm{d} \nu \geq-2\left(1+C_{r}\right) \sqrt{\left(1+\omega^{2}\right) \sigma^{\prime}(\omega)} .
$$

Considering the integral (A.5) as the sum of the two integrals over $\mathcal{A}_{ \pm}$and using the estimates (A.7) and (A.12) we get

$$
\lim _{\eta \rightarrow 0^{+}} \Re \mathrm{e} \tilde{\kappa}^{\prime}(\omega+\mathrm{i} \eta) \geq A-2\left(1+C_{r}\right) \sqrt{\left(1+\omega^{2}\right) \sigma^{\prime}(\omega)} .
$$

Therefore, $\left|\Re \mathrm{e} \kappa^{\prime}(\omega)\right| \geq \max \left(0, A-2\left(1+C_{r}\right) \sqrt{\left(1+\omega^{2}\right) \sigma^{\prime}(\omega)}\right)$ and together with (A.4) it follows that

$$
\begin{aligned}
& \left|\kappa^{\prime}(\omega)\right|^{2}+\Im \mathrm{m} \kappa(\omega) \\
\geq & \pi\left(1+\omega^{2}\right) \sigma^{\prime}(\omega)+\left|\Re \mathrm{e} \kappa^{\prime}(\omega)\right|^{2} \\
\geq & \pi\left(1+\omega^{2}\right) \sigma^{\prime}(\omega)+\left(\max \left\{0, A-2\left(1+C_{r}\right) \sqrt{\left(1+\omega^{2}\right) \sigma^{\prime}(\omega)}\right\}\right)^{2} \\
\geq & \underbrace{\frac{\pi A^{2}}{4\left(1+C_{r}\right)^{2}+\pi}}_{:=C_{2}},
\end{aligned}
$$

where in the last inequality we estimated the minimum of the quadratic function $\rho \rightarrow A^{2}-4 A\left(1+C_{r}\right) \rho+\left(4\left(1+C_{r}\right)^{2}+\pi\right) \rho^{2}$. 